San Jose State University

SJSU ScholarWorks

Doctoral Projects

Master's Theses and Graduate Research

Spring 4-2017

\title{
Efficacy of Continuous Passive Motion after Total Knee Arthroplasty in Veteran Patients
}

Jaimee Riza B. Hare

California State University, Northern California Consortium Doctor of Nursing Practice

Follow this and additional works at: https://scholarworks.sjsu.edu/etd_doctoral

Part of the Other Nursing Commons, and the Perioperative, Operating Room and Surgical Nursing Commons

\section{Recommended Citation}

Hare, Jaimee Riza B., "Efficacy of Continuous Passive Motion after Total Knee Arthroplasty in Veteran Patients" (2017). Doctoral Projects. 57.

DOI: https://doi.org/10.31979/etd.c95j-wtz9

https://scholarworks.sjsu.edu/etd_doctoral/57

This Doctoral Project is brought to you for free and open access by the Master's Theses and Graduate Research at SJSU ScholarWorks. It has been accepted for inclusion in Doctoral Projects by an authorized administrator of SJSU ScholarWorks. For more information, please contact scholarworks@sjsu.edu. 


\section{ABSTRACT \\ EFFICACY OF CONTINUOUS PASSIVE MOTION AFTER TOTAL KNEE ARTHROPLASTY IN VETERAN PATIENTS}

Since the early 1980s, continuous passive motion has been used as adjunct therapy in patients' rehabilitation following total knee arthroplasty. Although existing literature challenged the benefits of continuous passive motion claiming it had no added short-term or long-term benefits after knee arthroplasty (Boese et al., 2014; Chen et al., 2012; Herbold et al., 2014; Leach et al., 2006; Maniar et al., 2012), the existing literature is difficult to generalize and apply to the veteran population at San Francisco Veterans Affairs Medical Center. This study was undertaken to determine the efficacy of continuous passive motion in helping veteran patients at San Francisco Veterans Affairs Medical Center achieve post-operative range of motion goals following total knee arthroplasty. Using a retrospective design an extensive chart review was conducted. Analysis of Variance (ANOVA) with time as a repeated measure independent variable, continuous passive motion as a between-subjects independent variable, and extension/flexion as dependent variables was conducted. For extension, time was statistically significant $(p<.001)$ but mean extension did not differ between continuous passive motion and no continuous passive motion $(p=.976)$. Similarly, for flexion, time was statistically significant $(p<.001)$ but mean flexion did not differ between continuous passive motion and no continuous passive motion $(p=.128)$. Therefore, this research is consistent with current literature, which claims that continuous passive motion does not have short-term benefits, particularly in the area of range of motion. 
EFFICACY OF CONTINUOUS PASSIVE MOTION AFTER TOTAL KNEE ARTHROPLASTY IN VETERAN PATIENTS

by

Jaimee Riza B. Hare

\author{
A project \\ submitted in partial \\ fulfillment of the requirements for the degree of \\ Doctor of Nursing Practice \\ California State University, Northern Consortium \\ Doctor of Nursing Practice
}

April 2017 


\section{APPROVED \\ For the California State University, Northern Consortium Doctor of Nursing Practice:}

We, the undersigned, certify that the project of the following student meets the required standards of scholarship, format, and style of the university and the student's graduate degree program for the awarding of the master's degree.

Jaimee Riza B. Hare

Project Author

Colleen O'Leary-Kelley, $\mathrm{PhD}, \mathrm{RN}$ (Chair)

Nursing

Betty King, MSN, APRN, CCRN, FNP-BC

San Francisco Veterans Affairs Medical Center

January Tenorio, MS, APRN, CCNS

San Francisco Veterans Affairs Medical Center 


\section{AUTHORIZATION FOR REPRODUCTION}

\section{OF DOCTORAL PROJECT}

JBH I grant permission for the reproduction of this project in part or in its entirety without further authorization from me, on the condition that the person or agency requesting reproduction absorbs the cost and provides proper acknowledgment of authorship.

Permission to reproduce this project in part or in its entirety must be obtained from me. 


\section{ACKNOWLEDGMENTS}

I want to give special thanks to my project chairperson, Dr. Colleen O’LearyKelley, for her support, advice, and editing eye; my project committee members, Betty King and January Tenorio for their support and encouraging words;

Dr. Alexis Dang for his support and serving as my principal investigator to this capstone project; to my friends and family, most especially my children Chelsea and Alexander who continue to motivate and inspire me to dream big; and finally, to our nation's veterans, thank you for your service. 
TABLE OF CONTENTS

Page

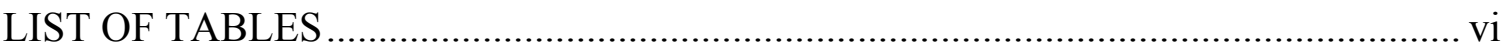

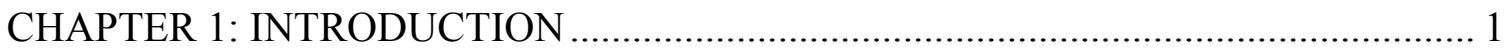

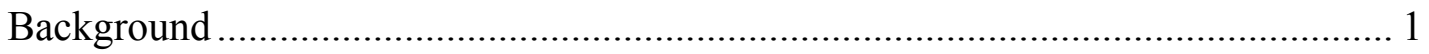

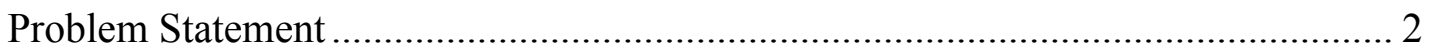

Purpose

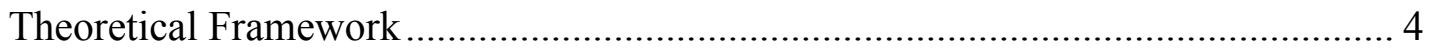

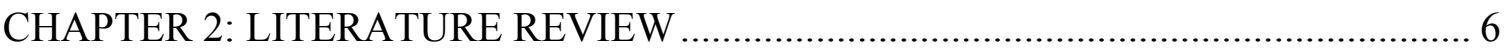

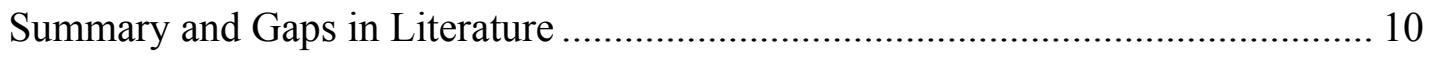

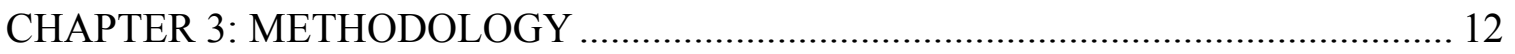

Research Design and Study Setting .................................................................... 12

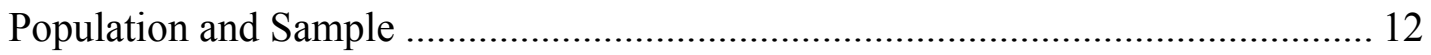

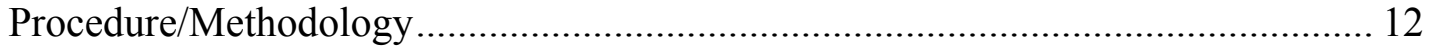

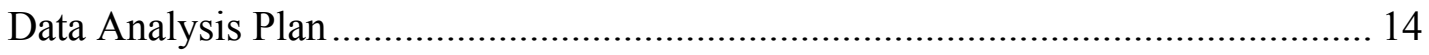

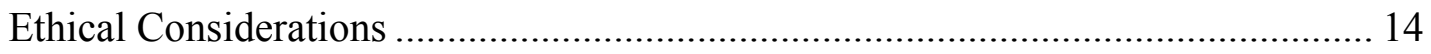

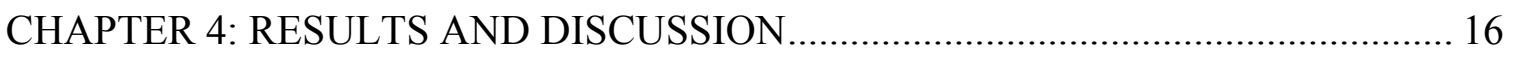

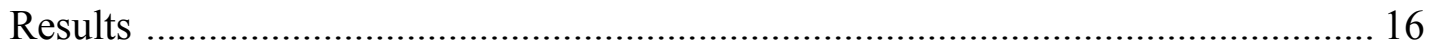

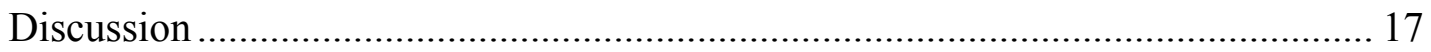

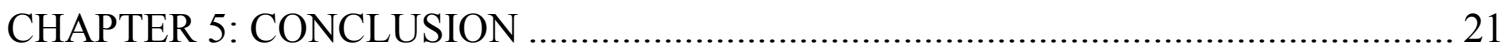

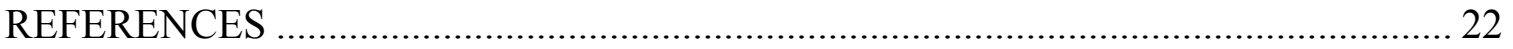




\section{LIST OF TABLES}

Page

Table 1. Range of Motion (Extension) Descriptive Statistics ...................................... 25

Table 2. Range of Motion (Flexion) Descriptive Statistics ....................................... 25

Table 3. Test of Within-Subjects Effects Measuring for Extension …………………... 26

Table 4. Test of Within-Subjects Effects Measuring for Flexion ……............................. 26

Table 5. Pairwise Comparisons of Extension and Time ……………………………..... 26

Table 6. Pairwise Comparisons of Flexion and Time …………………………......... 27 


\section{CHAPTER 1: INTRODUCTION}

Since the early 1980s, continuous passive motion has been used as adjunct therapy in patients' rehabilitation following total knee arthroplasty. Although existing literature challenged the benefits of continuous passive motion claiming it had no added short-term or long-term benefits after knee arthroplasty (Boese et al., 2014; Chen et al., 2012; Herbold et al., 2014; Leach et al., 2006; Maniar et al., 2012), the existing literature is difficult to generalize and apply to the veteran population at San Francisco Veterans Affairs Medical Center. This study was undertaken to determine the efficacy of continuous passive motion in helping veteran patients at San Francisco Veterans Affairs Medical Center achieve post-operative range of motion goals following total knee arthroplasty.

\section{Background}

Osteoarthritis is a debilitating and degenerative joint disease characterized by joint line narrowing, bone remodeling, bony osteophytes, and ligamentous laxity (Litwic, Edwards, Dennison, \& Cooper, 2013). Almost 50\% of Americans 75 years old and older suffer from osteoarthritis (Litwic et al., 2013). Total knee arthroplasty, which is a surgical procedure, can alleviate pain, knee instability, deformity, and improve the quality of life of persons with osteoarthritis (Matassi, Duerinckx, Vandenneucker, \& Bellemans, 2014). According to Centers for Disease Control and Prevention (2014) 454,652 total knee replacements were performed in the United States in 2004. The success of a total knee arthroplasty is largely dependent on post-operative rehabilitation. Patients need to regain range of motion necessary to perform functional activities such as walking and climbing stairs. Failure to meet post-operative range of motion goals results in knee stiffness, 
which limits a patient's ability to perform functional activities and places the patient at risk for additional interventions (Yeoh et al., 2012).

At San Francisco Veterans Affairs Medical Center, post-operative rehabilitation following total knee arthroplasty includes inpatient, home, and outpatient physical therapy, and less recently the distribution of a continuous passive motion device. The continuous passive motion device is a motorized device that passively flexes and extends the knee through a pre-determined range of motion (Lenssen et al., 2008). It was thought to help patients achieve their range of motion goals quicker. The device was delivered to patients' homes within a few days of hospital discharge and patients were instructed on how to operate it. Patients were given a two-week rental.

In May 2015, the orthopedic surgery department made the decision to discontinue distribution of continuous passive motion devices for two reasons, cost and current literature. A two-week rental would cost the organization $\$ 350$. Current literature claimed no added short-term or long-term benefits after knee arthroplasty. This will be discussed in detail in the literature review section.

\section{Problem Statement}

Since distribution of continuous passive motion devices was discontinued at San Francisco Veterans Affairs Medical Center, 10 patients from May 2015 to June 2016 failed to meet their post-operative range of motion goals. As such, these patients had to undergo additional intervention to manipulate the knee under anesthesia to break through adhesions and scar tissue. Additional intervention means more cost to the organization. The biggest risk of a manipulation under anesthesia is the risk of supracondylar femur fracture due to the pressure applied (Wied et al., 2015). Additionally, patients have 
voiced disappointment over the device's discontinuation as many of them attest to its efficacy. A study was needed to determine if continuous passive motion was indeed effective in helping post-operative total knee arthroplasty veteran patients achieve range of motion goals, thus decreasing the need for manipulations under anesthesia.

\section{Purpose}

The existing literature is difficult to generalize and apply to the veteran population at San Francisco Veterans Affairs Medical Center. To close the gaps in literature, this study was undertaken. The aim of this study was to answer the following questions:

(1) Is continuous passive motion effective in helping post-operative total knee arthroplasty veteran patients achieve range of motion goals?

(2) Comparing patients that received continuous passive motion after discharge to those that did not, what are patients' range of motion measurements before surgery, upon hospital discharge, and at their first post-operative visit?

The hypothesis was that continuous passive motion helped veteran patients meet their post-operative range of motion goals after total knee arthroplasty. Range of motion goals are $0^{\circ}$ extension and $>90^{\circ}$ degrees flexion by patients' first post-operative visit, generally six weeks after surgery. Additionally, it was hypothesized that patients that received continuous passive motion would have better range of motion measurements by their first post-operative visit compared to those that did not receive continuous passive motion.

This study is relevant to the orthopedic surgery and physical therapy departments as well as the patients that undergo total knee arthroplasty. Each year, the orthopedic surgery department performs roughly 520 joint replacement surgeries, about half of 
which are total knee arthroplasties. Veteran patients that have end-stage osteoarthritis and have failed conservative treatments, including nonsteroidal anti-inflammatory drugs, steroid injections, physical therapy, and knee bracing are good candidates for total knee arthroplasty.

\section{Theoretical Framework}

Hildegard Peplau's interpersonal relations theory, which emphasizes the nursepatient relationship, is widely used across all fields of nursing. Peplau's theory offers a framework for helping post-operative total knee arthroplasty veteran patients meet their range of motion goals, which was the focus of this study. The nurse-patient relationship has four important elements: nurse, patient, patient need, and nurse expertise. These are exemplified in the phases of the nurse-patient relationship. Initially, Peplau proposed three phases of the nurse-patient relationship: orientation, working, and resolution (Nelson, 2015). In later publications, a fourth phase, pre-orientation was added.

In the pre-orientation phase, the nurse gathers information about the patient and begins to explore his/her thoughts and feelings about the patient. In the orientation phase, the nurse and the patient meet and they begin to develop trust and rapport. Problems and issues are identified after questions are asked and issues are clarified. The working phase is where the most activity occurs. This phase is further divided into two subgroups: identification subphase and exploitation subphase. In identification subphase, specific problems are outlined. The nurse commits to work on these problems and the patient commits to work towards change. In the exploitation subphase, the patient tries new behaviors in a safe environment. Finally, in the resolution phase, the nurse-patient relationship terminates. The patient feels a sense of security because his/her needs were 
met (Nelson, 2015).

Over the course of several months, the nurse practitioners in the orthopedic surgery department and veteran patients progress through the four phases of the nursepatient relationship: pre-orientation, orientation, working, and resolution (Nelson, 2015). Nurse practitioners gather information about the patients by conducting chart reviews (pre-orientation). Nurse practitioners first meet the patients in the clinic or during their history and physical (orientation). Nurse practitioners then advance to the working phase, which starts when patients are admitted to the hospital post-operatively and continues with patients' follow-up visits. During these times, nurse practitioners educate patients on continuous passive motion, range of motion, and strengthening exercises. Nurse practitioners are their advocates when they have not received their continuous passive motion devices or when home health physical therapy has not contacted them. The relationship terminates when follow-up visits are no longer indicated (resolution). 
CHAPTER 2: LITERATURE REVIEW

Various databases, including EBSCO and PubMed were used to search for relevant literature on total knee arthroplasty. Terms such as "total knee arthroplasty," “continuous passive motion," and "manipulation under anesthesia" were used. There were no time or study design restrictions to the search. English only studies were used. Studies that compared continuous passive motion with alternative forms of range of motion rehabilitation devices and exercises were selected for review.

Mau-Moeller et al. (2014) conducted a randomized active-controlled clinical study to examine the efficacy of sling therapy and continuous passive motion after total knee arthroplasty. The control group had physiotherapy and continuous passive motion and the intervention group had physiotherapy and sling therapy. The study took place in an inpatient hospital setting. There were 38 participants, 19 in the intervention group (mean age 68.8, 63.2\% male and 36.8\% female) and 19 in the control group (mean age $67.1,53.6 \%$ male and $46.4 \%$ female). The primary outcome measured was range of motion. Range of motion was measured using a goniometer, which had substantial intertester and intra-tester reliability according to their literature review. Using unpaired student's $t$ test and ANCOVA, they found that flexion was significantly higher by six degrees $\left(95 \%\right.$ CI 0.9 to $\left.11.2^{\circ} ; p=.022\right)$ in the sling therapy group post-operatively. This showed that sling therapy had a clinically relevant beneficial short-term effect on flexion compared to continuous passive motion.

Boese et al. (2014) conducted a prospective, randomized, experimental study that examined the effects of early aggressive continuous passive motion and fixed flexion proceeding progressive continuous passive motion on short-term outcomes compared to 
standardized physical therapy alone. They had three groups: 1) continuous passive motion device on and moving from immediate post-operative period, 2) continuous passive motion device on and stationary at 90 degrees flexion for the first night and then moving throughout the rest of their stay, and 3) no continuous passive motion. The study took place in a community hospital. They had adequate sample sizes in the three groups ( $n=50,51,54$, respectively). Sample demographics were fairly equal between the three groups with mean ages of 69.1, 66.7, and 68.3 (respectively) and percentage of males being $30.9 \%, 29.4 \%$, and $38.9 \%$ (respectively). The primary outcome measured was range of motion. It was not mentioned how range of motion was measured or if the measurement tool was tested for reliability and validity. Using MANOVA to analyze their data, they found no significant test differences between the groups for any variable. For final range of motion, measurements were 109.6, 109, and 109.5 for the three groups, respectively $(p=.96)$. They found that continuous passive motion provided no apparent benefit to patients recovering from total knee arthroplasty in all outcome variables.

Maniar, Baviskar, Singhi, and Rathi (2012) conducted a prospective, randomized study examining the effects of using continuous passive motion. They had three groups: 1) no continuous passive motion, 2) one day no continuous passive motion, and 3) three day no continuous passive motion. The study took place in an inpatient hospital. Eightfour participants were evenly divided into the three groups. Mean ages for the three groups were $67.42,66.81$, and 66.06 , respectively. Male and female ratios for the three groups were $2: 26,3: 25$, and $4: 26$, respectively. The primary outcome measured was range of motion, which was measured using a goniometer. They did not state whether the goniometer was tested for reliability or validity. Demographic and clinical characteristics 
of the subjects and baseline measurements were compared between groups by use of ANOVA for continuous variables and $\mathrm{x}^{2}$ for categorical data. A nonparametric KruskalWallis test was used when the data were not normally distributed. Student $t$ test was used for comparison of data within groups. Average range of motion for all three groups at day three was $69.8^{\circ}$, at day five was $85.2^{\circ}$, at day fourteen was $94.9^{\circ}$, at day forty-two was $109.7^{\circ}$, and at day ninety was $117.5^{\circ}$. They reported no statistical significance. Improvement in range of motion was likely attributable to the physical therapy they received. They did not include tables or $p$ values for range of motion in the article. They found that continuous passive motion offered no benefit in immediate functional recovery post-total knee arthroplasty.

Chen et al. (2012) conducted a prospective, controlled study examining the effects of continuous passive motion on range of motion in patients undergoing total knee arthroplasty within six months post-surgery. The study took place at Kaohsiung Medical University Hospital in Taiwan. There were 107 participants, 68 in the treatment group (mean age $69.25 \pm 6.79$ ) and 39 in the control group (mean age $69.46 \pm 8.17$ ). The primary outcome measured was range of motion. Range of motion was measured using a goniometer, but reliability and validity were not mentioned. A Mann-Whitney test was used to analyze the efficacy between study groups and repeated ANOVA was used to examine the differences at various time points. They found no significant difference in range of motion between groups at the end of six months (treatment group 125-51 \pm 5-99 and control group 125-13 $\pm 6-44(p=.81))$. They found that aggressive continuous passive motion did not provide obvious benefits in range of motion.

Leach, Reid, and Murphy (2006) conducted a prospective randomized single- 
blind study with a one-year follow-up to examine the influence of continuous passive motion use on flexion and extension. The setting was not mentioned. Eighty-five participants were recruited and 82 participants were randomly assigned to either the treatment or control group ( $n=38$ and $n=44$, respectively). Mean ages for groups were 71.2 and 72.9 , respectively. The control group did not receive continuous passive motion and the treatment group did. The primary outcome measured was range of motion. Range of motion was measured using a goniometer, but reliability and validity were not mentioned. Measurements were taken before surgery, at discharge, six weeks, six months, and twelve months post-operatively. After using a two-sample $t$-test, they found no significant difference between groups. The $p$ values for Wilcoxon rank sum tests were $p=.44$ preoperatively, $p=.19$ at discharge, $p=.48$ at six weeks, $p=.56$ at six months and $p=.40$ at one year. They found that continuous passive motion use following total knee arthroplasty did not influence range of motion.

Herbold et al. (2014) conducted a randomized study examining the effects of using a continuous passive motion device for individuals with poor range of motion after total knee arthroplasty. The setting was a rehabilitation facility. Participants $(n=141)$ were randomly assigned to either the control or treatment group. The control group ( $n=$ 71) received three hours of therapy each day and the treatment group $(n=70)$ received the same therapy plus two hours of continuous passive motion each day. The primary outcome measured was range of motion. Range of motion was measured using a goniometer, which had good inter-tester reliability for knee flexion (intraclass correlation coefficient .89-.98) and fair to good for knee extension (intraclass correlation coefficient, .64-.92). Descriptive analysis and ANCOVAs of discharge knee flexion and extension 
active range of motion were conducted. Mean flexion active range of motion in the continuous passive motion group was $83+/-10$, and the control group not using the continuous passive motion was $86+/-7.9$ at discharge, revealing no significant difference $\left(\mathrm{F}_{1,138}=3.1\right.$, mean square error $\left.=81.38, p<.08\right)$. Continuous passive motion use in postacute rehabilitation did not appear to offer long-term benefits after unilateral total knee arthroplasty, regardless of initial range of motion.

\section{Summary and Gaps in Literature}

Since the early 1980 s, continuous passive motion has been used as adjunct therapy in patients' rehabilitation following total knee arthroplasty. Two biggest reported benefits were that it helped patients to meet range of motion goals and reduced the need for manipulations (Boese et al., 2014). However, these benefits were challenged as the literature showed that continuous passive motion had no added short-term or long-term benefits after knee arthroplasty (Boese et al., 2014; Chen et al., 2012; Herbold et al., 2014; Leach et al., 2006; Maniar et al., 2012). In fact, in most of the studies continuous passive motion was discontinued as part of their rehabilitation protocols (Boese et al.; Herbold et al.; Leach et al.; Maniar et al.).

The existing literature is difficult to generalize and apply to the veteran population at San Francisco Veterans Affairs Medical Center since different continuous passive motion and rehabilitation protocols were used. A couple of studies (Maniar et al., 2012; Mau-Moeller et al., 2014) did not have adequate sample sizes and Herbold et al. (2014) did not have pre-operative range of motion measurements.

To close the gaps in literature, this project had a sample size of 220 patient records. It had pre-operative measurements and post-operative measurements up until six 
weeks post-discharge. All patients adhered to the same post-operative rehabilitation protocol. Most of all, the study applied specifically to the veteran population at San Francisco Veterans Affairs Medical Center. 
CHAPTER 3: METHODOLOGY

\section{Research Design and Study Setting}

A retrospective design was used to address the research questions. The study took place at San Francisco Veterans Affairs Medical Center. The Veterans Health Administration provides comprehensive care to our nation's veterans. Veterans Health Administration consists of 150 medical centers and San Francisco Veterans Affairs Medical Center is among them (United States Department of Veterans Affairs, 2015). San Francisco Veterans Affairs Medical Center offers a variety of healthcare services to veterans living in northern California.

\section{Population and Sample}

Subjects were patient records of veteran patients who underwent a primary total knee arthroplasty at San Francisco Veterans Affairs Medical Center from January 2015 to June 2016. The sample size was 220 . In order be to considered for this study patients needed to have had a primary total knee arthroplasty at the San Francisco Veterans Affairs Medical Center from January 2015 to June 2016. All age groups and genders were included. Patients that expired prior to their first post-operative visit were excluded. Patients that had partial knee arthroplasties, revisions, or had complications (e.g. infection) were also excluded. Since this was a retrospective study using extensive chart review for data collection, informed consent was not needed. There was no direct patient interaction.

\section{Procedures/Methodology}

Data were collected through extensive chart review by this researcher. Data collection started when Institutional Review Board approval was obtained from both San 
Francisco Veterans Affairs Medical Center and California State University Fresno in July 2016. Data collection continued through August 2016. Range of motion data were determined using provider reported measurements. Using a retrospective approach, this researcher compared range of motion (extension and flexion) measurements on patients that received continuous passive motion in their homes for the first two weeks after hospital discharge with those patients that did not. An extensive chart review was performed to determine range of motion measurements (extension and flexion) at specific time periods (i.e. pre-operatively, at discharge, and at the six-week follow-up visit). San Francisco Veterans Affairs Medical Center uses Veterans Health Information Systems and Technology Architecture's (vistA) Computerized Patient Record System (CPRS) for its electronic health record. CPRS was used for the extensive chart review.

Data were collected from January 2015 to June 2016. This researcher gathered demographic information (i.e. age and gender) from the cover sheet. Date of surgery/admission and date of discharge were collected in order to determine length of stay. Additionally, disposition information (i.e. home versus skilled nursing facility) was collected. Charts of patients who expired prior to their first post-operative visit, all partial knee arthroplasties, revisions, and any complications (e.g. infection) were excluded from chart review. Surgical reports, progress notes from physical therapists and orthopedic surgery providers (i.e. residents, attending physicians, physician assistants, clinical nurse specialist, and nurse practitioners) were reviewed for flexion and extension measurements. Surgical reports were reviewed for the surgeon's name and prosthetic implant brand. Prosthetic consult orders were reviewed to determine whether patients were provided with a continuous passive motion device. Finally, follow-up visit progress 
notes were reviewed to determine if the patient required a manipulation under anesthesia. Data were collected and complied in an excel worksheet. No data collection instrumentation was used. Once all the data were collected for a particular patient, the chart was re-reviewed for accuracy and completeness.

\section{Data Analysis Plan}

To evaluate whether continuous passive motion was effective in helping postoperative total knee arthroplasty veteran patients achieve range of motion goals, range of motion was compared between patients that received continuous passive motion and those that did not. Range of motion measurements were examined before surgery, upon hospital discharge and at patients' first post-operative visit. This researcher worked with a statistician to analyze the data. In addition to descriptive statistics, ANOVA with time as a repeated measure independent variable, continuous passive motion as a betweensubjects independent variable, and extension/flexion as dependent variables were performed.

\section{Ethical Considerations}

Because this was a retrospective study, there were no study interventions or direct contact with patients. The biggest potential risk was violation of patients' privacy and confidentiality. Every effort was made to protect patient information while their data were used as part of this study. To maintain patient confidentiality, identifiable patient information was coded. Identifiable patient information was kept electronically and within the San Francisco Veterans Affairs Medical Center's computer firewall. Personal identification card and password were required to log onto San Francisco Veterans Affairs Medical Center's computer system. Data were stored and destroyed according to 
San Francisco Veterans Affairs Medical Center and California State University Fresno Institutional Review Boards' policies. 


\section{CHAPTER 4: RESULTS AND DISCUSSION}

\section{Results}

The sample size consisted of majority males 204 (92.7\%) versus 16 females (7.3\%). Of the 220 patient records reviewed, 63 (28.6\%) patients received continuous passive motion and 157 (71.4\%) patients did not. Eleven patients (5\%) required additional intervention to manipulate the knee under anesthesia. Tables 1 and 2 illustrate mean extension and flexion measurements for those that received continuous passive motion: pre-operative $(3.27,112.45)$, hospital discharge $(5.27,77.25)$, and first postoperative visit $(3.27,98.77)$. Mean extension and flexion measurements for those that did not receive continuous passive motion were as follows: pre-operative $(3.10,113.75)$, hospital discharge $(5.30,78.03)$, and first post-operative visit $(3.44,103.76)$.

ANOVA was conducted with time as a repeated measure independent variable, continuous passive motion as a between-subjects independent variable, and extension as the dependent variable. In the test of within-subjects effects in table 3, the effect of time (i.e. pre-operative, hospital discharge, and first post-operative visit) on extension (dependent variable) was examined. Time was statistically significant $(p<.001)$ but there was no interaction between time and continuous passive motion $(p=.921)$. The HuynhFeldt p-value corrected for any violations of the repeated measures in ANOVA's assumption of sphericity. In the test of between-subjects, mean extension did not differ between continuous passive motion and no continuous passive motion $(p=.976)$.

The same tests were conducted for flexion. In the test of within-subjects effects in table 4, the effect of time (i.e. pre-operative, hospital discharge, and first post-operative visit) on flexion (second dependent variable) was examined. Time was statistically 
significant $(p<.001)$ but there was no interaction between time and continuous passive motion ( $p=.293$ ). The Huynh-Feldt $\mathrm{p}$-value corrected for any violations of the repeated measures in ANOVA's assumption of sphericity. In the test of between-subjects, mean flexion did not differ between continuous passive motion and no continuous passive motion $(p=.128)$.

Finally, as shown in table 5, to determine which time point the mean extension differed, the pairwise comparison table was reviewed where time one is pre-operative, time two is hospital discharge, and time three is first post-operative visit. Time one versus time two differed $(p<.001)$, time one versus time three did not differ $(p=.702)$, and time two versus time three differed $(p<.001)$. As shown in table 6 , to determine which time point the mean flexion differed, the pairwise comparison table was reviewed. All pairwise comparisons (time one versus time two, time one versus time three, and time two versus time three) were statistically significant $(p<.001)$.

\section{Discussion}

This study aimed to answer the following questions:

(1) Is continuous passive motion effective in helping post-operative total knee arthroplasty veteran patients achieve range of motion goals?

(2) Comparing patients that received continuous passive motion after discharge to those that did not, what are patients' range of motion measurements before surgery, upon hospital discharge, and at their first post-operative visit? 
To answer the first question, this study showed that continuous passive motion is not effective in helping post-operative total knee arthroplasty veteran patients achieve range of motion goals. Patients that were given continuous passive motion device rentals for two weeks after hospital discharge did not have any better range of motion compared to those that did not receive this device.

To answer the second question, mean extension and flexion measurements for patients that received continuous passive motion were as follows: pre-operative (3.27, 112.45), hospital discharge $(5.27,77.25)$, and first post-operative visit $(3.27,98.77)$. Mean extension and flexion measurements for those that did not receive continuous passive motion were as follows: pre-operative $(3.10,113.75)$, hospital discharge $(5.30$, 78.03), and first post-operative visit $(3.44,103.76)$.

\section{Limitations}

One limitation is the possible threat instrumentation had to internal validity. Different providers (i.e. attending physicians, residents, nurse practitioners, clinical nurse specialist, and physical therapists) obtained range of motion measurements. There can be observer changes, meaning that one provider might measure extension at three degrees whereas another provider might measure five degrees. Even if providers received the same training and used the same measurement tool, it is difficult to eliminate this variability. Therefore, this is an unavoidable limitation and will be reported when the study is submitted for publication. Because this was a retrospective study, there was no opportunity for random sampling, which could have strengthened this study. Rather, a 
method called convenience sampling was used. For this study, patients' charts were reviewed for data if the following criteria were met: veteran patient at San Francisco Veterans Affairs Medical Center, underwent primary total knee arthroplasty from January 2015 to June 2016, and did not have a partial knee arthroplasty, revision, or any complications (e.g. infection). Another limitation is the possibility of confounding, or the presence of other risk factors that were not measured. Finally, retrospective studies cannot determine causation, only association.

\section{Implications for Nursing Practice}

This project will impact nursing in several ways. Nurses (e.g. registered nurses, licensed vocational nurses, nurse practitioners, and clinical nurse specialists) provide care to the post-operative total knee arthroplasty patient. Nurses provide education on postoperative rehabilitation. Since continuous passive motion devices were discontinued, nurses educate patients on current literature, which indicate that continuous passive motion does not have any added short-term or long-term benefits. Nurses educate and reinforce range of motion (e.g. active range of motion) and strengthening exercises. Finally, nurses can conduct further studies in this area.

This project has several limitations as mentioned above. The next logical research study would address these limitations. Ideally, future studies in this area should be a prospective study with random sampling and an adequate sample size. Additionally, there are many other methods used to help post-operative total knee arthroplasty patients regain their range of motion. Other methods may include physical therapy and sling therapy. 
Future studies can compare range of motion measurements using the different methods. 


\section{CHAPTER 5: CONCLUSION}

In closing, since the early 1980s, continuous passive motion has been used as adjunct therapy in patients' rehabilitation following total knee arthroplasty. Existing literature challenged the benefits of continuous passive motion claiming it had no added short-term or long-term benefits after knee arthroplasty (Boese et al., 2014; Chen et al., 2012; Herbold et al., 2014; Leach et al., 2006; Maniar et al., 2012). This study was undertaken to determine the efficacy of continuous passive motion in helping veteran patients at San Francisco Veterans Affairs Medical Center achieve post-operative range of motion goals following total knee arthroplasty. Using a retrospective design an extensive chart review was conducted. ANOVA with time as a repeated measure independent variable, continuous passive motion as a between-subjects independent variable, and extension/flexion as dependent variables was conducted. For extension, time was statistically significant $(p<.001)$ but mean extension did not differ between continuous passive motion and no continuous passive motion $(p=.976)$. Similarly, for flexion, time was statistically significant $(p<.001)$ but mean flexion did not differ between continuous passive motion and no continuous passive motion $(p=.128)$. Therefore, this research is consistent with current literature, which claims that continuous passive motion does not have short-term benefits, particularly in the area of range of motion. Given that the San Francisco Veterans Affairs Medical Center's current practice of not distributing continuous passive motion devices aligns with current literature and this research, the recommendation is that this organization continues current practice. 


\section{REFERENCES}

Boese, C. K., Weis, M., Phillips, T., Lawton-Peters, S., Gallo, T., \& Centeno, L. (2014). The efficacy of continuous passive motion after total knee arthroplasty: A comparison of three protocols. The Journal of Arthroplasty, 29(6), 1158-1162.

Centers for Disease Control and Prevention (2014). Arthritis-related statistics. Retrieved from http://www.cdc.gov/arthritis/data_statistics/arthritis_related_stats.htm

Chen, L., Chen, C., Lin, S., Chien, S., Su, J. Y., Huang, C. Y., . . Huang. H. (2012). Aggressive continuous passive motion exercise does not improve knee range of motion after total knee arthroplasty. Journal of Clinical Nursing, 22, 389-394. doi: $10.1111 / \mathrm{j} .1365-2702.2012 .04106 . x$

Herbold, J. A., Bonistall, K., Blackburn, M., Agolli, J., Gaston, S., Gross, C., . . Babyar, S. (2014). Randomized controlled trial of the effectiveness of continuous passive motion after total knee replacement. Archives of Physical Medicine and Rehabilitation, 95, 1240-1245. doi: http://dx.doi.org/10.1016/j.apmr.2014.03.012

Leach, W., Reid, J., \& Murphy, F. (2006). Continuous passive motion following total knee replacement: A prospective randomized trial with follow up to 1 year. Knee Surgery, Sports Traumatology, Arthroscopy, 14, 922-926. doi: 10.1007/s00167006-0042-9

Lenssen, T. A. F, van Steyn, M. J. A., Crijns, Y. H. F., Waltje, E. M. H., Roox, G. M., Geesink, R. J. T., . . De Bie, R. A. (2008). Effectiveness of prolonged used of continuous passive motion (CPM), as an adjunct to physiotherapy, after total knee 
arthroplasty. BMC Musculoskeletal Disorders, 9, 60-70. doi:10.1186/1471-24749-60

Litwic, A., Edwards, M., Dennison, E., \& Cooper, C. (2013). Epidemiology and burden of osteoarthritis. Br Med Bull, 105, 185-199. doi:10.1093/bmb/lds038.

Maniar, R. N., MS, Baviskar, J. V., Singhi, T. \& Rathi, S. S. (2012). To use or not to use continuous passive motion post-total knee arthroplasty. The Journal of Arthroplasty, 27, 193-200. doi:10.1016/j.arth.2011.04.009

Matassi, F., Duerinckx, J., Vandenneucker, H., \& Bellemans, J. (2014). Range of motion after total knee arthroplasty: The effect of a preoperative home exercise program. Knee Surg Sports Traumatol Arthrosc, 22, 703-709. doi: 10.1007/s00167-0122349-z.

Mau-Moeller, A., Behrens, M., Finze, S., Bruhn, S., Bader, B., \& Mittelmeier, W. (2014). The effect of continuous passive motion and sling exercise training on clinical and functional outcomes following total knee arthroplasty: A randomized activecontrolled clinical study. Health and Quality of Life Outcomes, 12, 68. doi:10.1186/1477-7525-12-68

Nelson, S. (2015). Theories focused on interpersonal relationships. In J. B. Butts \& K. L. Rich (Eds.), Philosophies andtheories for advanced nursing practice (pp.257305). Burlington, MA: Jones and Bartlett Learning.

U.S. Department of Veterans Affairs (2015). About VHA. Retrieved from http://www.va.gov/health/aboutVHA.asp 
Wied, C., Thomsen, M. G., Kallemose, T., Myhrmann, L., Jensen, L. S., Husted, H., \& Troelsen, A. (2015). The risk of manipulation under anesthesia due to unsatisfactory knee flexion after fast-track total knee arthroplasty. The Knee, 22, 419-423. doi: http://dx.doi.org/10.1016/j.knee.2015.02.008

Yeoh, D., Nicolaou, N., Goddard, R., Willmott, H., Miles, K., East, D., . . . ButlerManuel, A. (2012). Manipulation under anesthesia post total knee replacement: Long term follow up. The Knee, 19, 329-331. doi:10.1016/j.knee.2011.05.009 


\begin{tabular}{|c|c|c|}
\hline \multicolumn{3}{|l|}{ Table 1} \\
\hline \multicolumn{3}{|c|}{ Range of Motion (Extension) Descriptive Statistics } \\
\hline & Continuous Passive Motion & Mean, $\mathrm{N}$ \\
\hline \multirow[t]{2}{*}{ Pre-operative Extension } & No & $3.10,131$ \\
\hline & Yes & $3.27,60$ \\
\hline \multirow[t]{2}{*}{ Discharge Extension } & No & $5.30,131$ \\
\hline & Yes & $5.27,60$ \\
\hline Post-operative Visit & No & $3.44,131$ \\
\hline Extension & Yes & $3.27,60$ \\
\hline \multicolumn{3}{|l|}{ Table 2} \\
\hline \multicolumn{3}{|c|}{ Range of Motion (Flexion) Descriptive Statistics } \\
\hline & Continuous Passive Motion & Mean, $\mathrm{N}$ \\
\hline \multirow[t]{2}{*}{ Pre-operative Extension } & No & $113.75,135$ \\
\hline & Yes & $112.45,60$ \\
\hline \multirow[t]{2}{*}{ Discharge Extension } & No & $78.03,135$ \\
\hline & Yes & $77.25,60$ \\
\hline Post-operative Visit & No & $103.76,135$ \\
\hline Extension & Yes & $102.23,60$ \\
\hline
\end{tabular}


Table 3

Test of Within-Subjects Effects Measuring for Extension

\section{Huynh-Feldt Significance}

Time

0.0

Time*Continuous Passive Motion 0.921

Table 4

Test of Within-Subjects Effects Measuring for Flexion

Huynh-Feldt Significance

Time $\quad 0.0$

Time*Continuous Passive Motion 0.293

Table 5

Pairwise Comparisons of Extension and Time

\begin{tabular}{llccr}
\hline Time & Time & Mean Difference & Std. Error & Significance \\
Pre-operative & Hospital & -2.099 & .463 & .000 \\
& Discharge & & & \\
& First Post- & -.172 & .449 & .702 \\
& Operative Visit & & & \\
Hospital & Pre-operative & 2.099 & .463 & .000 \\
Discharge & & & & \\
& & & .391 & .000
\end{tabular}


Operative Visit

$\begin{array}{lllll}\text { First Post- } & \text { Pre-operative } & .172 & .449 & .702\end{array}$

Operative Visit

$\begin{array}{llll}\text { Hospital } & -1.927 & .391 & .000\end{array}$

Discharge

Table 6

Pairwise Comparisons of Flexion and Time

$\begin{array}{llccr}\text { Time } & \text { Time } & \text { Mean Difference } & \text { Std. Error } & \text { Significance } \\ \text { Pre-operative } & \text { Hospital } & 11.834 & 1.315 & .000 \\ & \text { Discharge } & & & \\ & \text { First Post- } & 35.459 & 1.549 & .000 \\ & \text { Operative Visit } & & & \end{array}$

$\begin{array}{lllll}\text { Hospital } & \text { Pre-operative } & -11.834 & 1.315 & .000\end{array}$

Discharge

$\begin{array}{lll}\text { First Post- } & 23.625 & 1.524\end{array}$

Operative Visit

$\begin{array}{lllll}\text { First Post- } & \text { Pre-operative } & -35.459 & 1.549 & .000\end{array}$

Operative Visit

$\begin{array}{lll}\text { Hospital } & -23.625 & 1.524\end{array}$

Discharge 